\title{
artigo
}

Oliveira, S.S.W.; Amaral, V.R.S.; Vasconcelos, R.S.; Rocha, E.S.; Sá, K.N.;

O uso da prática espiritual no tratamento da dor de pessoas com câncer

\section{O uso da prática espiritual no tratamento da dor de pessoas com câncer}

The use of spiritual practice in the treatment of cancer person pain

Or use of spiritual practice does not treatment of pessoas com câncer

\begin{abstract}
RESUMO
Objetivo: Identificar se os pacientes com câncer utilizam a prática espiritual no enfrentamento da dor física causada pelo câncer e quais são as estratégias espirituais utilizadas. Método: Trata-se de estudo transversal e descritivo, realizado em um hospital de referência em tratamento quimioterápico e uma casa de apoio a pessoas com câncer, localizados no sul da Bahia, entre agosto e setembro de 2019. Para a coleta foi utilizado um questionário com variáveis sociodemográficas e perguntas acerca da espiritualidade. Resultados: A maioria das pessoas realizavam estratégias espirituais: oração, leitura da bíblia, ir à missa/culto, hinos de louvor, acender velas, rezar o terço, meditação e ter fé nos médicos e em Deus. Conclusão: 0 uso da prática espiritual é uma estratégia utilizada pelas pessoas com câncer que participaram do estudo para o alívio da dor física causada pela doença, e que tal estratégia reduz a dor.
\end{abstract}

DESCRITORES: Neoplasias; Dor do Câncer; Espiritualidade.

\section{ABSTRACT}

Objective: To identify whether cancer patients use spiritual practice to cope with the physical pain caused by cancer and what spiritual strategies are used. Method: This is a cross-sectional and descriptive study, conducted at a referral hospital for chemotherapy treatment and a cancer support center, located in southern Bahia, between August and September 2019. For the collection, a questionnaire was used. with sociodemographic variables and questions about spirituality. Results: Most people pursued spiritual strategies: prayer, bible reading, attending mass/worship, hymns of praise, lighting candles, praying the rosary, meditation, and having faith in physicians and God. Conclusion: The use of spiritual practice is a strategy used by people with cancer who participated in the study to relieve the physical pain caused by the disease, and that such a strategy reduces pain.

DESCRIPTORS: Neoplasms; Cancer Pain; Spirituality.

\section{RESUMEN}

Objetivo: Identificar si los pacientes con cáncer utilizan la práctica espiritual para hacer frente al dolor físico causado por el cáncer y qué estrategias espirituales se utilizan. Método: Este es un estudio transversal y descriptivo, realizado en un hospital de referencia para tratamiento de quimioterapia y un centro de apoyo para el cáncer, ubicado en el sur de Bahía, entre agosto y septiembre de 2019. Para la recolección, se utilizó un cuestionario. con variables sociodemográficas y preguntas sobre espiritualidad. Resultados: La mayoría de las personas siguieron estrategias espirituales: oración, lectura de la Biblia, asistir a misa/adoración, himnos de alabanza, encender velas, rezar el rosario, meditar y tener fe en los médicos y en Dios. Conclusión: El uso de la práctica espiritual es una estrategia utilizada por personas con cáncer que participaron en el estudio para aliviar el dolor físico causado por la enfermedad, y que dicha estrategia reduce el dolor. DESCRIPTORES: Neoplasias; Dolor de Cáncer; Espiritualidad.

RECEBIDO EM: 23/12/2019 APROVADO EM: 24/12/2019

\section{Sharon Shyrley Weyll Oliveira}

Enfermeira. Bacharel em Direito. Mestre em Enfermagem pela Universidade Federal da Bahia. Docente do Curso de Enfermagem da Universidade Estadual de Santa Cruz. Discente do Doutorado em Medicina e Saúde Humana pela Escola Bahiana de Medicina e Saúde Pública. ORCID: https://orcid.org/0000-0002-3388-9710.

\section{Verônica Rabelo Santana Amaral}

Enfermeira. Mestre em Ciências da Saúde pela Universidade Estadual do Sudoeste da Bahia. Especialista em Saúde Coletiva pela Universidade Federal do Sul da Bahia. ORCID: https://orcid.org/0000-0003-1657-0254.

\section{Rayzza Santos Vasconcelos}

Enfermeira. Mestranda em Ciências da Saúde pela Universidade Estadual de Santa Cruz. Especialista em Saúde Coletiva pela Universidade Federal do Sul da Bahia. Especialista em Enfermagem em Dermatologia pela Faculdade Futura. ORCID: https:/orcid.org/0000-0001-9276-3731. 


\section{Evely Rocha Lima}

Farmacêutica. Mestre em Ciências da Saúde pela Universidade Estadual do Sudoeste da Bahia. ORCID: https://orcid.org/00000002-8989-3313.

\section{Katia Nunes Sá}

Fisioterapeuta. Pós-doutora em Ciência da Informação e Comunicação Científica pelo Instituto Brasileiro de Informação em Ciência e Tecnologia. Docente da Pós-Graduação, nível doutorado, da Escola Bahiana de Medicina e Saúde Pública. ORCID: https://orcid.org/0000-0002-0255-4379.

\section{INTRODUÇÃO}

A s neoplasias representam no Brasil a segunda causa de mortalidade, devido a este dado o câncer é uma condição cercada por medos e incertezas ${ }^{(1)}$. Diante da perspectiva de final de vida, as pessoas com câncer podem passam por estágios no seu processo de aceitação da doença: negação (não acredita nos exames e na equipe de saúde); raiva (possui sentimentos de ira, revolta, e ressentimento); barganha (realiza promessas para o prolongamento da vida ou redução/cessação da dor ou males físicos); depressão (sente tristeza aliada a outros sentimentos); aceitação (aceita a doença e o tratamento) $)^{(2)}$.

Neste contexto, o câncer é visto como uma condição que está associada ao sofrimento e à morte, portanto no momento do diagnóstico, as pessoas se deparam com sentimentos negativos (aflição, ansiedade, depressão, desesperança e agressividade), esse contexto necessita de redução da sobrecarga emocional, utilizando estratégias de enfrentamento para obter o reequilíbrio psíquico ${ }^{(3)}$.

Dentre as estratégias de enfrentamento, é comum que os pacientes com câncer adotem estratégias religiosas e espirituais para lidar com o estresse, na tentativa de aliviar o sofrimento e melhorar a esperança. Embora distintos, a espiritualidade e a religiosidade estão interligadas, pois a espiritualidade consiste na busca do ser humano pelo significado da vida, contemplando os aspectos ligados à natureza, cultura, sociedade, dentre outros; já a religiosidade se caracteriza pelo segmento de normas e princípios doutrinários definidos por uma entidade, com atitudes de devoção, crença e esforço para viver religiosamente ${ }^{(4)}$.

A espiritualidade auxilia as pessoas em condiçôes de vulnerabilidade a sobreviver com a dor e as situações cotidianas, através da ressignificação das experiências que vi$\mathrm{vem}^{(5)}$. A partir da contextualização acerca da temática, elencaram-se as seguintes questões norteadoras: A espiritualidade se apresenta como estratégia de enfrentamento da dor física em pacientes com câncer? Quais práticas espirituais são utilizadas? O objetivo deste trabalho foi identificar se os pacientes com câncer utilizam a prática espiritual no enfrentamento da dor física causada pelo câncer e quais são as estratégias espirituais utilizadas.

\section{Dentre as estratégias}

de enfrentamento,

é comum que os pa-

cientes com câncer

adotem estratégias

religiosas e espirituais

para lidar com o es-

tresse, na tentativa de

aliviar o sofrimento e

melhorar a esperança.

\section{METODOLOGIA}

Este estudo é um recorte do projeto inti- tulado "A espiritualidade no enfrentamento da dor em pacientes oncológicos" aprovado pelo Comitê de Ética em Pesquisa da Universidade Estadual de Santa Cruz, sob o parecer n. ${ }^{\circ} 3.022 .500$ por meio do CAAE 01564218.2.0000.5526. Trata-se de estudo transversal e descritivo, realizado em um hospital de referência em tratamento quimioterápico e uma casa de apoio a pessoas com câncer, localizados no sul da Bahia, entre agosto e setembro de 2019.

A população do estudo no hospital foram as pessoas internadas com o diagnóstico de câncer e, na casa de apoio, foram as pessoas em tratamento do câncer que estavam hospedas no período da coleta. Os critérios de inclusão foram: pessoas com diagnóstico de câncer, com idade maior ou igual 18 anos, que relataram o diagnóstico de câncer e que aceitaram participar do estudo mediante assinatura do Termo de Consentimento Livre e Esclarecido (TCLE). Os critérios de exclusão foram: ausência de dor atual ou pregressa, diagnóstico de doença mental e impossibilidade ou dificuldade de comunicação.

Para a coleta, foi utilizado um questionário com variáveis sociodemográficas e perguntas acerca da espiritualidade (A sua religiosidade interferiu no enfrentamento da sua doença?; Você realiza alguma prática espiritual? Se sim, qual?; Você acha que a sua prática espiritual reduz a dor física causada pela doença?), que foi aplicado por duas enfermeiras treinadas. As pessoas internadas no hospital foram entrevistadas individualmente no leito, e na casa de apoio foram abordadas durante as atividades educacionais e direcionadas para a entrevista.

Os dados foram digitados e analisados pelo software Statistical Package for the Social Sciences, versão 21.0. Foi realizada a análise descritiva, sendo calculadas as frequências absoluta e relativa. 


\section{RESULTADOS}

Das 60 pessoas com câncer selecionadas, 39 foram excluídas por não atenderem aos critérios do estudo, das quais 17 não apresentaram dor atual ou pregressa, 14 não relataram o diagnóstico de câncer e 08 se encontravam impossibilitadas ou com dificuldade de comunicação no momento da coleta. Dessa forma, foram incluídas 21 pessoas, observando-se que houve predominância do sexo feminino e a idade média apresentada foi de 51,1 anos. Em relação a situação conjugal, 12 $(57,1 \%)$ relataram não ter companheiro, 13 (61,9\%) viviam no domićlíio com uma a duas pessoas e $11(52,4 \%)$ tinham renda familiar de um a três salários.

Em relação ao perfil clínico, 05 (23,8\%) tinham câncer de mama, 03 (14,3\%) no colo de útero, 02 (9,5\%) no pulmão, 02 (9,5\%) no estômago, 02 (9,5\%) na cabeça e pescoço, $02(9,5 \%)$ no esôfago, 01 $(4,8 \%)$ na próstata, $01(4,8 \%)$ na bexiga, $01(4,8 \%)$ no ouvido, $01(4,8 \%)$ no reto e 01 (4,8\%) mieloma múltiplo.

Sobre o perfil religioso, $20(95,2 \%)$ pessoas possuíam religião e, destas, 10 (50\%) se declararam católicas, 09 (45\%) evangélicas e 01 (5\%) judaica. Verificou-se que a maioria dos pacientes relataram que a religiosidade auxiliou no enfrentamento do câncer 18 (58,7\%). Quanto à espiritualidade, a maioria das pessoas realizavam estratégias espirituais 20 (95,2\%), sendo elas: oração, leitura da bíblia, ir à missa/ culto, hinos de louvor, acender velas, rezar o terço, meditação e ter fé nos médicos e em Deus. Dentre as pessoas que realizavam estratégias espirituais, 16 (80\%) afirmaram que a espiritualidade reduziu a dor física causada pelo câncer.

\section{DISCUSSÃO}

A prática espiritual no tratamento da dor das pessoas com câncer foi apresentada como uma estratégia capaz de reduzir a dor física causada pelo câncer na população em estudo. A espiritualidade ocorre através da fé, que está relacionada à esperança de um conforto, é uma fonte de
A prática espiritual

no tratamento da

dor das pessoas

com câncer foi

apresentada como

uma estratégia capaz

de reduzir a dor

física causada pelo

câncer na população

em estudo. A

espiritualidade

ocorre através da fé,

que está relacionada

à esperança de um

conforto, é uma

fonte de apoio que

possibilita crer

em algo positivo,

reduzindo a

ansiedade e os

$\operatorname{medos}^{(6)}$. apoio que possibilita crer em algo positivo, reduzindo a ansiedade e os medos ${ }^{(6)}$.

A fé é um processo interior que pode ou não conter uma busca religiosa ${ }^{(7)}$. Neste estudo, apenas uma pessoa relatou não ter religião. A maioria das pessoas com câncer utilizam a religião como ferramenta de enfrentamento, sobretudo, em situações adversas, a religião é um item necessário para melhorar o convívio com o câncer, aliviar os impactos provocados e melhorar o conhecimento ${ }^{(8)}$. A doença e a proximidade com a morte convocam as pessoas a se aproximar de um ser superior em busca da cura, o tratamento deixa de ser uma atribuição exclusivamente médica, e passa a ser direcionada à uma cura mágica, que se relaciona à religião ${ }^{(9)}$.

A religiosidade auxiliou no enfrentamento do câncer para a grande parte dos participantes dessa pesquisa, que além de estarem lidando com a doença, tinham como agravo a dor relacionada ao câncer. A dor está presente em aproximadamente $30 \%$ das pessoas com câncer, sendo considerada o quinto sinal vital, portanto é indispensável sua avaliação periódica, a fim de oferecer condições adequadas para o manejo da doença e garantir a qualidade de vida dos pacientes ${ }^{(10)}$.

O tratamento da dor vai além de medidas farmacológicas, sendo a espiritualidade uma estratégia utilizada por muitos pacientes, particularmente aqueles com doenças graves, e os pacientes dependem dela como um mecanismo de enfrentamento positivo ${ }^{(11)}$. A espiritualidade foi utilizada por grande maioria dos participantes desta pesquisa, sendo relatada como uma ferramenta relevante capaz de reduzir a dor física causada pelo câncer.

Segundo Sousa et $\mathrm{al}^{(12)}$, as pessoas com câncer recorrem à espiritualidade para encontrar um sentido para esta experiência de adoecimento, tendo em vista que ela ativa os transmissores desencadeando uma sensação de bem-estar, melhora do sistema imune, diminui o estresse e a ansiedade. A espiritualidade ocorre por meio de atividades que buscam fortalecer o significado da vida, a fé ou componentes existenciais, a paz consigo mesmo e com os outros, agrupadas 
com o termo estratégias espirituais ${ }^{(13)}$.

Os achados do presente estudo, quanto ao uso de estratégias espirituais, confirmam a ideia de que elas apoiam e transmitem forças às pessoas. Assim como em Arrieira et $\mathrm{al}^{(14)}$, a fé em Deus e a leitura da bíblia foram utilizadas como prática espiritual neste estudo. A fé representa uma força propulsora, atuando como ponto positivo no enfrentamento da doença, tem a capacidade de explicar aquilo que aparenta ser inexplicável durante as situações adversas e aos momentos difíceis e de tristezas vivenciadas pelo adoecimento ${ }^{(15)}$.

A principal ou a segunda estratégia de enfrentamento usada para lidar com a dor é a oração, conforme foi revelado nos estudos de Koenig ${ }^{(16)}$ e Rippentrop ${ }^{(17)}$. A efetividade da prece, através de uma oração cristã sem invocação de santos ou santidades, mostrou-se positiva na redução de ansiedade de pacientes com câncer em tratamento de quimioterapia, para tanto as pesquisadoras utilizaram como intervenção a citação do salmo 138 da Bíblia Sagrada, que trata sobre a onisciência divina: Deus tudo sabe e tudo vế(18).

A religiosidade e a espiritualidade são práticas eficazes que auxiliam na ressignificação da trajetória de pessoas com câncer, aliviando o sofrimento em situações difíceis e estressantes ${ }^{(7)}$. Assim, os aspectos religiosos/espirituais devem ser compreendidos pelos profissionais de saúde dian- te do processo de adoecimento de uma pessoa com câncer.

\section{CONCLUSÃO}

Os achados do presente estudo corroboram com o conhecimento sobre o uso da prática espiritual em pessoas com câncer, pois evidenciam que a espiritualidade é uma estratégia utilizada por pessoas com câncer para o alívio da dor física causada pela doença, e que tal estratégia reduz a dor. Neste estudo, a espiritualidade esteve interligada à religiosidade, pois as estratégias espirituais predominantemente utilizadas pelas pessoas com câncer estudadas foram de caráter religiosas.

\section{REFERÊNCIAS}

1. Malta DC, Moura L de, Prado RR do, Escalante JC, Schmidt MI, Duncan BB. Mortalidade por doenças crônicas não transmissíveis no Brasil e suas regiões, 2000 a 2011. Epidemiol Serv Saúde. 2014;23(4):599-608.

2. Kübler-Ross E. Sobre a morte e o morrer. 9. ed. Fontes M, organizador. São Paulo; 2008. 304 p.

3. Feldman RS. Introdução à Psicologia. 10. ed. Artmed, organizador. Porto Alegre; 2015. 656 p.

4. Matos TD de S, Meneguin S, Ferreira M de L da S, Miot HA. Qualidade de vida e coping religioso-espiritual em pacientes sob cuidados paliativos oncológicos. Rev Latino-Am Enferm. 2017;25:e2910.

5. Benites AC, Neme CMB, Santos MA Dos. Significados da espiritualidade para pacientes com câncer em cuidados paliativos. Estud Psicol. 2017;34(2):269-79.

6. Nobre C de FC, Mendes F. A experiência da pessoa com dor oncológica na sua transcendência. Rev Ibero-Americana Saúde e Envelhec. 2018;4(1):1224-39.

7. Ribeiro GS, Campos CS, Anjos ACY dos. Espiritualidade e religião como recursos para o enfrentamento do câncer de mama. Rev Pesqui Cuid é Fundam Online. 1 de julho de 2019;11(4):849-56.

8. Menezes RR, Kameo SY, Valença T dos S, Mocó GAA, Santos JM de J. Qualidade de vida relacionada à saúde e espiritualidade em pessoas com câncer. Rev Bras Cancerol. 2018;64(1):9-17.

9. Reis CG da C dos, Farias CP, Quintana AM. O vazio de sentido: suporte da Religiosidade para pacientes com câncer avançado. Psicol Ciência e Profissão. 2017;37(1):106-18.

10. Castro CC de, Pereira AK da S, Bastos BR. Implementation of the evaluation of pain as the fifth vital sign. J Nurs
UFPE online. 2018;12(11):3009-14.

11. Stewart WC, Adams MP, Stewart JA, Nelson LA. Review of clinical medicine and religious practice. Vol. 52, Journal of religion and health. 2013. p. 91-106.

12. Sousa FF de PR, Freitas SMF de M, Farias AGDS, Cunha M da CDSO, Araújo MFM de, Veras VS. Enfrentamento religioso/espiritual em pessoas com câncer em quimioterapia: revisão integrativa da literatura. SMAD Rev Eletrônica Saúde Ment Álcool e Drog (Edição em Port. 2017;13(1):45.

13. Phenwan T, Peerawong T, Tulathamkij K. The meaning of spirituality and spiritual well-being among thai breast cancer patients: a qualitative study. Indian J Palliat Care [Internet]. 2019;25(1):119-23.

14. Arrieira ICDO, Thoferhn MB, Schaefer OM, Fonseca AD da, Kantorski LP, Cardoso DH. O sentido do cuidado espiritual na integralidade da atenção em cuidados paliativos. Rev Gaúcha Enferm. 2017;38(3):e58737.

15. Linard AG, Silva FADRM da S. Mulheres submetidas a tratamento para câncer de colo uterino - percepção de como enfrentam a realidade. Rev Bras Cancerol. 2002;48(4):4938.

16. Koenig GH. Religion and medicine IV: religion, physical health, and clinical implications. Int J Psychiatry Med. 2001;31(3):321-36.

17. Rippentrop AE. A review of the role of religion and spirituality in chronic pain populations. Rehabil Psychol. 2005;50(3):278.

18. Carvalho CC, Chaves $\mathrm{E}$ de $\mathrm{CL}$, lunes $\mathrm{DH}$, Simão $\mathrm{TP}$, Grasselli C da SM, Braga CG. Effectiveness of prayer in reducing anxiety in cancer patients. Rev da Esc Enferm. 2014;48(4):684-90. 